\title{
Integration of Control Design and Variable Geometry Suspension Construction for Vehicle Stability Enhancement
}

\author{
Balázs Németh and Péter Gáspár
}

\begin{abstract}
The paper proposes a method in which the construction of the variable geometry suspension system and the design of robust suspension control are performed simultaneously in order to enhance vehicle stability. In the control scheme a predefined road trajectory required by the driver with a steering command is followed to carry out various maneuvers. The variable geometry suspension system provides the modification of the mechanical geometry efficiently. During maneuvers an autonomous control system influences the camber angles of the rear wheels by using a variable geometry suspension system. The control system must guarantee various crucial vehicle performances such as trajectory tracking, roll stability and geometry limits. Since there is an interaction between the construction design and the control design a balance must be achieved between them.
\end{abstract}

\section{INTRODUCTION AND MOTIVATION}

The paper focuses on the construction design of a variable geometry suspension system. The suspension determines such critical components as the height of the roll center and half track change. The advantages of the variable geometry suspension are the simple structure, low energy consumption and low cost compared to other mechanical solutions such as an active front wheel steering. Since various safety and economy properties of the vehicle are determined by the suspension geometry it has significant influence on the control design. The control input is the camber angles of the rear wheels, with which the driver is supported to perform the various vehicle maneuvers, such as a sharp cornering.

Several papers for various kinematic models of suspension systems have been published. A review of the first variable geometry systems was presented by [1]. The control system varied the leverage ratio between the spring/damper unit and the road wheel assembly. A nonlinear model of the Macpherson strut suspension system was published by [2]. By using this model the kinematic parameters such as camber, caster and king-pin angles were examined. The kinematic design of a double-wishbone suspension system was examined by [3]. Seeking to meet the performance requirements often leads conflict situations and requires a compromise considering the kinematic and dynamic properties, see [4]. The vehicle handling characteristics based on a variable roll center suspension was proposed by [5]. A rear-suspension active toe control for the enhancement of driving stability was proposed

P. Gáspár and B. Németh are with Systems and Control Laboratory, Computer and Automation Research Institute, Hungarian Academy of Sciences, Kende u. 13-17, H-1111 Budapest, Hungary. E-mail: [gaspar; bnemeth] @sztaki.hu

The research was supported by the Hungarian National Office for Research and Technology through the project "Innovation of distributed driver assistance systems for a commercial vehicles platform" (TECH_08_2/22008-0088) which is gratefully acknowledged. by [6], [7]. The main focus on these methods is on the construction solution and the control design has received little attention. However, besides performances, the control design must handle important tasks such as disturbance attenuation and robustness against uncertainties.

In the paper robust LPV (Linear Parameter Varying) methods are proposed for control design. Based on the LPV modeling approaches the highly nonlinear effects can be considered in the state space description. Furthermore this state space representation of the LPV model is valid in the entire operating region of interest. The advantage of LPV methods is that the controller meets robust stability and nominal performance demands in the entire operational interval, since the controller is able to adapt to the current operational conditions, [8], [9].

This paper is organized as follows. Section II presents the control-oriented modeling of the tire and its formalization in lateral dynamics of vehicle model. In Section III the nonlinear variable geometry kinematic model of the suspension system is analyzed. The performances in the controloriented LPV model are presented in Section IV. In Section $\mathrm{V}$ the integration of the design of parameter-dependent LPV control and the construction of variable geometry suspension system is performed. Section VI illustrates the operation of the control system through different vehicle maneuvers.

\section{LATERAL DYNAMICS OF VEHICLE MODEL}

In the trajectory tracking control system the control input is the camber angle of the rear wheels. The model used in the control design is based on the bicycle model of the vehicle. In the model the wheel camber angle must also be taken into consideration. The Magic form of the tire dynamics describes the effect of the wheel camber angle on the lateral tire forces $\left(F_{y}\right)$, see [10]. The curves of $F_{y}$ via $\alpha$ as a function of wheel camber angle are illustrated by dashed line in Figure 1. Although the Magic form gives a highly accurate description of the tire model, a simplified form of the tire model is constructed for numerical reasons.

The main idea of the model construction is that the model approximates the Magic form in terms of small side-slip angles. The general Magic form is $F_{y}=$ $D_{y} \sin \left\{C_{y} \operatorname{atan}\left[B_{y} \alpha-E_{y}\left(B_{y} \alpha-\operatorname{atan}\left(B_{y} \alpha\right)\right)\right]\right\}$, where $B_{y}, C_{y}, D_{y}, E_{y}$ are parameters of the tire model and $\alpha$ is the wheel side-slip angle. In case of small side-slips the lateral force can be approximated in the following form: $F_{y}=C \alpha$ where $C$ is cornering stiffness and $\alpha$ is the tire side-slip angle. The parameter $B_{y}$ is extended with the following assumptions: $B_{y}=K_{y \alpha} /\left(C_{y} D_{y}+\varepsilon_{y}\right)$, where 


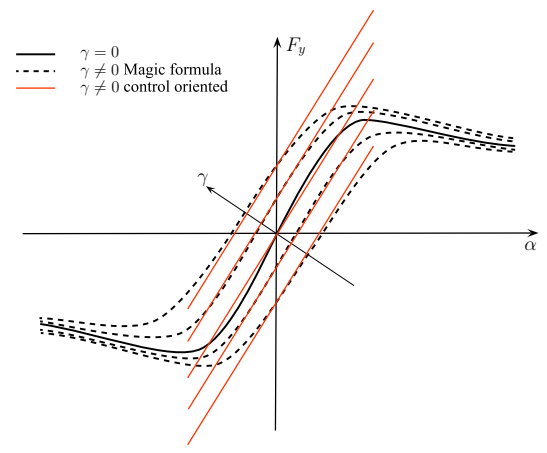

Fig. 1. Considering the wheel camber in the tire model

$K_{y \alpha}=K_{y \alpha 0}\left(1-p_{K y 3}(\sin \gamma)^{2}\right) \zeta_{3}$ in which $K_{y \alpha 0}$ depends on the vertical load of tire and $p_{K y 3}, \zeta_{3}, \varepsilon_{y}$ are parameters. The linearized tire model can be extended with the effect of wheel camber. It is approximated as a vertical offset of the $\gamma=0$ curve, see the curve illustrated by solid line in Figure 1. This control-oriented lateral tire model in the direction of the wheel ground contact velocity is approximated

$$
F_{y}=C \alpha+C_{\gamma} \gamma
$$

where $C_{\gamma}$ is a coefficient which represents the degree of offset and $\gamma$ is the wheel camber angle. Note that the second term occurs only in the rear wheels in the model.

The vehicle is moving in the entire plane of the road, thus both the longitudinal and the lateral dynamics must be taken into consideration as shown in Figure 2. The driver input of

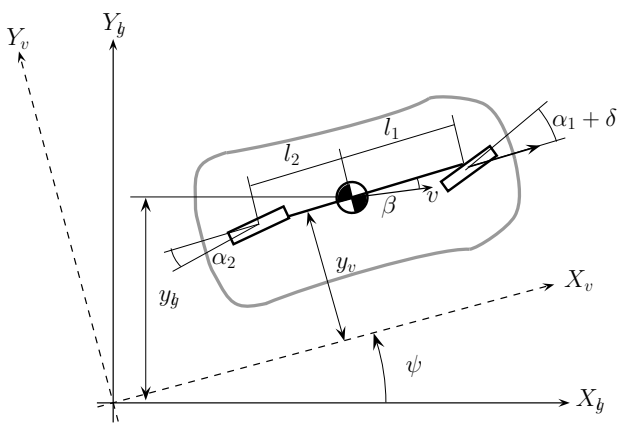

Fig. 2. Lateral model of the vehicle

the system is the front steering angle $\delta$, while the control input of the trajectory tracking system is the camber angle of the rear wheels $\gamma$. The longitudinal velocity is denoted by $v$.

The lateral dynamics of the vehicle is approximated by the bicycle model of the vehicle:

$$
\begin{aligned}
& J \ddot{\psi}=C_{1} l_{1} \alpha_{f}-C_{2} l_{2} \alpha_{r}-C_{2, \gamma} l_{2} \gamma \\
& m v(\dot{\psi}+\dot{\beta})=C_{1} \alpha_{f}+C_{2} \alpha_{r}+C_{2, \gamma} \gamma
\end{aligned}
$$

where $J$ is the yaw inertia of the vehicle, $l_{1}$ and $l_{2}$ are geometric parameters, $C_{1}$ and $C_{2}$ are cornering stiffnesses, $\psi$ is the yaw of the vehicle, $\beta$ is the side-slip angle. Moreover, $\alpha_{f}=-\beta+\delta-l_{1} \cdot \dot{\psi} / v$ and $\alpha_{r}=-\beta+l_{2} \cdot \dot{\psi} / v$ are the tire side slip angles at the front and rear, respectively. In the design of trajectory tracking control it is necessary to guarantee that the lateral position of the vehicle tracks the geometry of the road.

\section{Modeling of A VARIABle GeOMetry System}

In this section the kinematic model of the variable geometry mechanism based on the double wishbone suspension system is presented (see Figure 3). This model contains

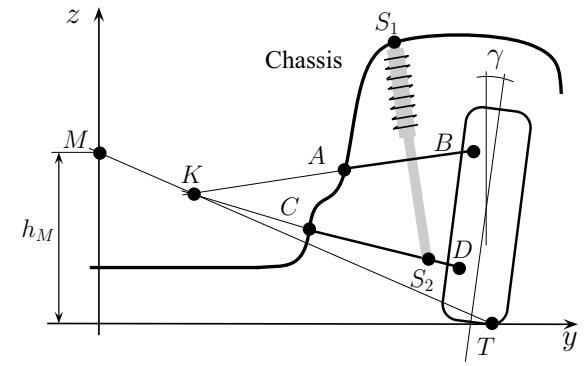

Fig. 3. Kinematic model of the suspension system

the geometry of the actuator and shows the suspension displacements. In this approach the masses, inertias and elasticity of the construction elements can be ignored. Thus the arms of the suspension are modeled as bar elements.

The suspension system is analyzed in a local coordinate system, whose center point is $C$. Point $A$ in the variable geometry system is able to move only in a horizontal direction. In the variable geometry suspension the change of point $A$ in the direction $y$ is the real input of the mechanism, which is denoted by $a_{y}$. Two further points $B$ and $D$ are marked on the tire, which moves both in directions $y$ and $z$. $T$ is the road-wheel contact point, which moves as a function of the road irregularities, $t_{y}, t_{z}$. The aim is to formalize the relationship between the input $a_{y}$ and the wheel camber output $\gamma$.

The coordinates of the points of suspension can be determined by using the following equations:

$$
\begin{aligned}
D_{y}^{2}+D_{z}^{2} & =L_{D C}^{2} \\
\left(D_{y}-B_{y}\right)^{2}+\left(D_{z}-B_{z}\right)^{2} & =L_{B D}^{2} \\
\left(A_{y}-B_{y}\right)^{2}+\left(A_{z}-B_{z}\right)^{2} & =L_{A B}^{2} \\
\left(D_{y}-T_{y}\right)^{2}+\left(D_{z}-T_{z}\right)^{2} & =L_{T D}^{2} \\
\left(B_{y}-T_{y}\right)^{2}+\left(B_{z}-T_{z}\right)^{2} & =L_{T B}^{2}
\end{aligned}
$$

where $\left(A_{y}, B_{y}, C_{y}, D_{y}, T_{y}, A_{z}, B_{z}, C_{z}, D_{z}, T_{z}\right)$ are the coordinates of suspension points in directions $y$ and $z$, $L_{A B}, L_{D C}$ are the lengths of arms, $L_{B D}, L_{T B}, L_{T D}$ are distances between points. The values of point coordinate A are divided into two parts: $A_{y}=\bar{A}_{y}+a_{y}$ and $A_{z}=\bar{A}_{z}+a_{z}$. Similarly, the values of the other point coordinates are also divided into two parts. The first part represents the constant nominal values of the suspension coordinates, i.e., the steady state position of the vehicle, while the second part shows the displacements of the suspension points. There are constrains on the suspension system, which are defined by $a_{z}=0$, $c_{y}=0$ and $c_{z}=0$. The output of the system is the camber of the wheel: $\gamma=\arccos \left\{\left(B_{z}-D_{z}\right) / L_{B D}\right\}$.

The goal of this model is to formalize the relationship between the actuator movement $a_{y}$ and the wheel camber. 
Using equation (3) the relationship is formalized between $a_{y}$ and the variables $\eta=\left[\begin{array}{lllll}b_{y} & b_{z} & d_{y} & d_{z} & t_{y}\end{array}\right]^{T}$. In the equation $t_{z}$ is the disturbance. Consequently, (3) is arranged into the following forms:

$$
\begin{aligned}
A_{\eta}(\eta) \eta & =K\left(t_{z}\right)+B_{\eta}\left(a_{y}\right) a_{y} \\
C_{\eta} \eta & =D_{\eta} .
\end{aligned}
$$

The vector $\eta$ is expressed from (4) as follows:

$$
\eta=A_{\eta}(\eta)^{-1}\left[K\left(t_{z}\right)+B_{\eta}\left(a_{y}\right) a_{y}\right]
$$

The input of the mechanism $a_{y}$ is expressed as follows:

$$
a_{y}=\left(C_{\eta} A_{\eta}(\eta)^{-1} B_{\eta}\left(a_{y}\right)\right)^{-1}\left[D_{\eta}-C_{\eta} A_{\eta}(\eta)^{-1} K\left(t_{z}\right)\right]
$$

It is a parameter-varying expression, which depends on $\eta$, $t_{z}$ and $a_{y}$. In this equation $\eta$ is unknown, the variables $A_{\eta}$, $D_{\eta}$, and $K\left(t_{z}\right)$ change as a function of $\eta$ and $t_{z}$. Moreover, $B_{\eta}$ depends on $a_{y}$. Consequently, the input of the variable geometry mechanism $a_{y}$ can only be computed by using an iterative procedure.

To solve this expression it is necessary to know the road excitation $t_{z}$ and choose accurate initial values for both $\eta_{0}$ and $a_{y 0}$. In the first step a new $\eta$ is determined based on (5). In the second step the input $a_{y}$ is computed based on (6). In the third step a mean square error is computed:

$$
\vartheta=\sum\left(\left(\eta-\eta_{0}\right)^{2}+\left(a_{y}-a_{y 0}\right)^{2}\right)
$$

The numerical solution of the problem is acceptable, if $\vartheta<$ $\varepsilon$, where $\varepsilon$ is a predefined small real number. Otherwise the re-iteration is performed by selecting $a_{y 0}=a_{y}$ and $\eta_{0}=\eta$.

In the following part of the section the effects of the input $a_{y}$ on vehicle components are analyzed. The relationship between $a_{y}$ and $\gamma$ as a function of $t_{z}$ based on the numerical solution of (6) is shown in Figure 4. An analysis shows that

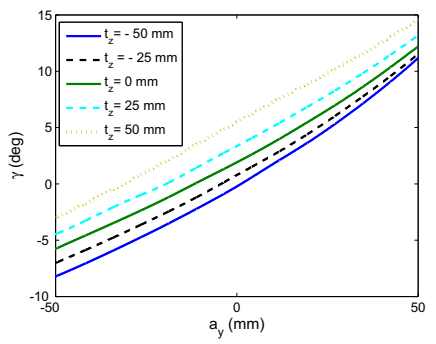

Fig. 4. $a_{y}-\gamma$ characteristics

it is possible to approximate the $a_{y}-\gamma$ curves with linear functions in the following form:

$$
\gamma=\kappa+\xi_{1} t_{z}+\varepsilon_{1} a_{y}
$$

It is noted that both wheels have the same static wheel camber on one axle. The static components of the lateral forces are approximately equal, thus in the next computations the constant $\kappa$ is omitted from (8).

The construction of suspension determines the height of the roll center of the chassis $\left(h_{M}\right)$. The roll center is determined by $A, B, C, D$ and $T$ points. The intersection of the arms $(A, B)$ and $(C, D)$ is marked by $K$. The intersection of the line $(T, K)$ and the vertical centerline of the chassis is the roll center itself. The relationship between $a_{y}$ and $h_{M}$ as a function of $t_{z}$ based on the numerical solution of (6) is shown in Figure 5. The height of the roll center can

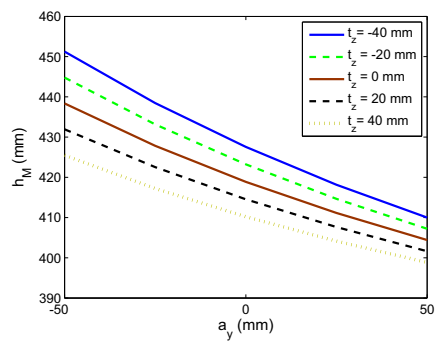

Fig. 5. Height of roll center

be divided into static and dynamic components as follows: $h_{M}=h_{M, s t}+\Delta h_{M}$. Component $h_{M, s t}$ represents the height of the roll center of a stationary vehicle, while $\Delta h_{M}$ represents the change of the height during travelling. The dynamic component is expressed in the following form:

$$
\Delta h_{M}=\xi_{2} t_{z}+\varepsilon_{2} a_{y}
$$

During travelling the half track change $(\Delta B)$ is also an important economical dynamic parameter of the suspension system, since it is related to tire wear. The relationship between $a_{y}$ and $\Delta B$ as a function of $t_{z}$ based on the numerical solution of (6) is shown in Figure 6. The figure

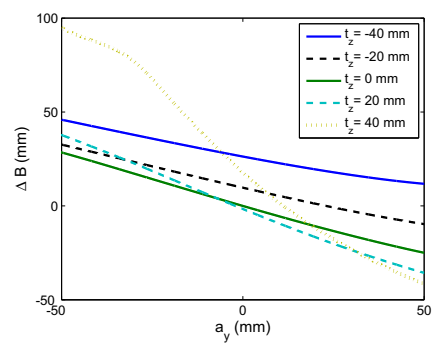

Fig. 6. Half track change

shows that although the relationship between $a_{y}$ and $\Delta B$ can be approximated linearly in low velocities, the nonlinear effects of the half track change increase in the high velocity domain. Thus the low and the high velocities are separated in the analysis. In the low velocity domain the following approximation is accepted: $-20 \mathrm{~mm} \leq t_{z} \leq 20 \mathrm{~mm}$ and $v<60 \mathrm{~km} / \mathrm{h}$. In this domain the suspension parameter $\Delta B$ is approximated in the following form:

$$
\Delta B=\xi_{3} t_{z}+\varepsilon_{3} a_{y}
$$

\section{PERFormances In THE CONTROL-ORIENTEd MODEL}

In the trajectory tracking control the lateral dynamics must be taken into consideration and the vehicle must follow the reference yaw-rate signal. The difference between the 
yaw-rate of the vehicle and the reference yaw-rate must be minimized:

$$
z_{1}=\left|\dot{\psi}_{\text {ref }}-\dot{\psi}\right| \rightarrow \min
$$

It is necessary to emphasize that the activation of wheel camber is important only at high velocities, since at low velocities in the urban traffic the driver usually does not require any assistance. In the high velocity domain the vehicle can reach instability at the critical velocity [10]: $v_{\text {crit }}=\sqrt{g\left(l_{1}+l_{2}\right) / \eta}$, where $\eta$ is the understeering coefficient. It suggests that the assistance of the rear wheel camber is particularly important around $v_{\text {crit }}$. A weighting function $W_{z 1}$ is applied in order to avoid the critical velocity, see Figure 7.

It has also shown that roll center depends on the controller actuation $\left(a_{y}\right)$ and $t_{z}$. The height of the roll center has an important role in the roll dynamics of the vehicle [11]:

$$
\left(I_{x x}+m \Delta h^{2}\right) \ddot{\phi}=m g \Delta h \phi+m v(\dot{\beta}+\dot{\psi})-B_{i} \sum_{i=1}^{4} F_{s u s p, i}
$$

where $\Delta h$ is the difference between the height of the center of gravity of the chassis and the roll center $\left(\Delta h=h_{C G}-\right.$ $\left.h_{M}\right), \phi$ is the chassis roll angle, $I_{x x}$ is the inertia of the chassis, $B_{i}$ is the half track and $F_{s u s p, i}$ are the vertical forces of suspension. In order to improve roll stability the height of the roll center $h_{M}$ must be minimized. Since $h_{M}$ is divided into two parts, i.e., $h_{M}=h_{M, s t}+\Delta h_{M}$, two performance criteria are formulated. According to the performance $z_{2}$ the difference between the roll center and the center of gravity must be minimized and according to the performance $z_{3}$ the dynamic displacement of the height of the roll center based on (9) must be minimized:

$$
\begin{aligned}
& z_{2}=\left|h_{C G}-h_{M, s t}\right| \rightarrow \min \\
& z_{3}=\left|\Delta h_{M}\right|=\left|\xi_{2} t_{z}+\varepsilon_{2} a_{y}\right| \rightarrow \min
\end{aligned}
$$

Equation of the roll dynamics shows, that the roll of the chassis depends on the velocity of the vehicle. Since the roll of the chassis is critical at high velocities, therefore the same assumption is used as in case of trajectory tracking.

During the control tasks it is necessary to prevent large control inputs. Therefore the fourth performance focuses on the minimum of the input displacement:

$$
z_{4}=\left|a_{y}\right| \rightarrow \min
$$

The control input is rather an economy parameter and not a safety parameter, thus it might be reduced in the high velocity domain. The selected weighting functions $W_{z 3}$ and $W_{z 4}$ are shown in Figure 7.

An additional important economy parameter is the half track change, because this parameter influences the tire wear. An average automobile drives the most of its lifespan in urban traffic, therefore it is necessary to guarantee economical operation at low velocities. Using a linear approximation and based on (10) the fifth performance criterion is formalized in the following form:

$$
z_{5}=|\Delta B|=\left|\xi_{2} t_{z}+\varepsilon_{3} a_{y}\right| \rightarrow \min
$$

The analysis shows that the half track change has nonlinear effects. Because of model uncertainties the half track change weighting function $W_{z 5}$ must be selected in such a way that performance $z_{5}$ has low values outside the validity range. The inadequacy of this performance can be compensated for with a sixth performance, which extends the half track change to the entire velocity range:

$$
z_{6}=\iint|\Delta B| d a_{y} d t_{z}
$$

It is also an economy performance, which must be taken into consideration at low velocities.

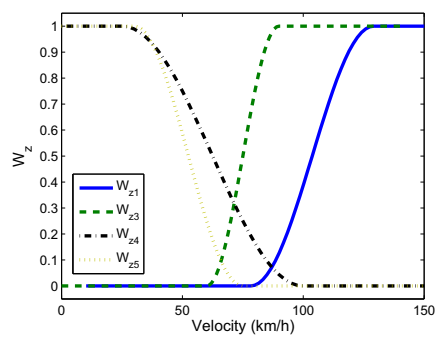

Fig. 7. Performance weights

The designed performances must be considered both in the control design and the construction design. In the control design the control input of the mechanism $a_{y}$ affects the performance specifications. In the construction design the selected points $B_{z}$ and $D_{z}$ play an important role, since these parameters influence most performances significantly. Therefore the integration of control design and suspension construction leads to an optimization task.

Performances can be divided into two groups. Performances $z_{1}, z_{3}, z_{4}$ and $z_{5}$ can be expressed by linear combinations of the control input $a_{y}$ and disturbance $t_{y}$. Vector $\mathcal{Z}_{1}$ includes the performances which are used in the control design:

$$
\mathcal{Z}_{1}=\left[\begin{array}{llll}
z_{1} & z_{3} & z_{4} & z_{5}
\end{array}\right]^{T}
$$

Performances $z_{2}$ and $z_{6}$ cannot be expressed by any linear combination and they are used only in the construction design. Vector $\mathcal{Z}_{2}$ includes the performances which are used in the construction design:

$$
\mathcal{Z}_{2}=\left[\begin{array}{ll}
z_{2} & z_{6}
\end{array}\right]^{T}
$$

\section{INTEGRATION OF CONTROL DESIGN AND SUSPENSION CONSTRUCTION}

The goal of this paper is to design the integration of the control design and the variable geometry suspension construction. First the design of a control system based on the performance $\mathcal{Z}_{1}$ is proposed and second the integrated design based on performances $\mathcal{Z}_{1}$ and $\mathcal{Z}_{2}$ is proposed. 


\section{Control design}

In Section II a lateral vehicle model is introduced, see (2). This model contains wheel camber $\gamma$ as control input, which can be replaced with $a_{y}$ according to (8). The motion equation of the vehicle is constructed in a state-space representation form:

$$
\dot{x}=A(\rho) x+B_{1}(\rho) w+B_{2}(\rho) u
$$

where the state vector of the system contains the yaw-rate and the side-slip angle $x=[\dot{\psi}, \beta]^{T}$. The control input of the system is $a_{y}$ on the rear wheels $u=a_{y}$, while front steering of the vehicle and $t_{z_{T}}$ are handled as the disturbances of the system $w=\left[\delta, t_{z}\right]^{T}$. The system matrices depend on the velocity of the vehicle nonlinearly. The velocity is assumed to be a measured signal. Using a scheduling variable $\rho=v$ the nonlinear model is transformed into an LPV model.

In the trajectory tracking problem a reference signal $\dot{\psi}_{\text {ref }}$ is introduced in order to guarantee the tracking of the road geometry. The control design is based on a weighting strategy, which is formalized through a closed-loop interconnection structure, see Figure 8. Input and output weighting functions are typically selected to the specifications of disturbances and the inverse of the specifications on the outputs.

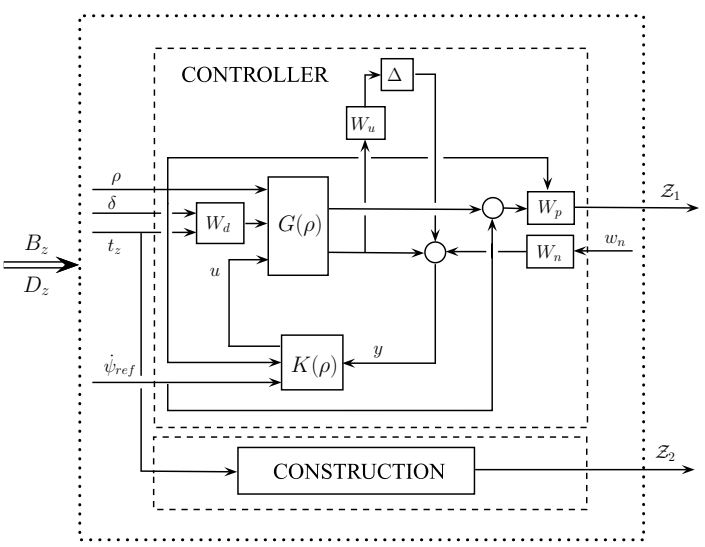

Fig. 8. Closed-loop interconnection structure

The control design is based on the LPV method that uses parameter-dependent Lyapunov functions, see [8], [9]. The quadratic LPV performance problem is to choose the parameter-varying controller in such a way that the resulting closed-loop system is quadratically stable and the induced $\mathrm{L}_{2}$ norm from the disturbance and the performances is less than a predefined value. The existence of a controller that solves the quadratic LPV performance problem can be expressed as the feasibility of a set of Linear Matrix Inequalities (LMIs), which can be solved numerically.

\section{Integrated design}

In the integrated design the construction of variable geometry suspension must also be designed. In the construction design the variables of the system $B_{z}$ and $D_{z}$ must be taken into consideration. It is important to emphasize that the control design and the construction design are not independent. The construction of the system influences the characteristics $\gamma\left(a_{y}, t_{z}\right), \Delta B$ and $\Delta h_{M}$, which are parts of the control design according to (17). The performances in (18) also affect the dynamics of the vehicle.

In the integrated design the operator norms from inputs of the system to the performance outputs $\mathcal{Z}_{1}$ have an essential role. $\mathcal{T}_{i, j}\left(B_{z}, D_{z}\right)$ is the operator, in which $i$ is the input and $j$ is the output of the system, see Figure 8. Weighting functions $W_{z, i}$ represent the importance of the performances of $\mathcal{Z}_{1}$. These features have been considered in the design of LPV control. In the following the construction components are taken into consideration. The analysis shows that the velocity influences both the lateral dynamics of the vehicle and performance weighting functions.

Step 1: In the first step the velocity domain is gridded for several points and all of the selected grid points the operator norms $\left\|\mathcal{T}_{i, j}\left(v, B_{z}, D_{z}\right)\right\|$ are computed. Since the priority of the different velocities is considered in the control design, it is possible to compute the algebraic average of the operator norms. Then the computed operator norms are summarized: $\left\|\mathcal{T}_{j}\left(B_{z}, D_{z}\right)\right\|=\sum_{i}\left\|\mathcal{T}_{i, j}\left(B_{z}, D_{z}\right)\right\|$. The result of these computations are indexes, which represent the fulfillment of the performance $\mathcal{Z}_{1}$ in a given suspension construction.

Step 2: In the second step it is possible to compute the performances of $\mathcal{Z}_{2}$ in a given construction. These performances must be normalized using a maximum value of the performances $z_{i, \max }: J_{z, k}\left(B_{z}, D_{z}\right)=\left|z_{k}\left(B_{z}, D_{z}\right)\right| / z_{k, \max }$. The result of these computations are also indexes, which represent the fulfillment of the $\mathcal{Z}_{2}$ performance at a given suspension construction.

Step 3: In the third step these performance indexes are summarized, which results in a global cost for the fulfillment of $\mathcal{Z}_{1}$ and $\mathcal{Z}_{2}$ performances in a given suspension construction:

$$
\mathcal{J}\left(B_{z}, D_{z}\right)=\sum_{j}\left\|\mathcal{T}_{j}\left(B_{z}, D_{z}\right)\right\|+\sum_{k} J_{z, k}\left(B_{z}, D_{z}\right) .
$$

It can be shown that the computed cost $\mathcal{J}\left(B_{z}, D_{z}\right)$ depends on the suspension construction. Finally, it is necessary to find the construction parameters $B_{z}$ and $D_{z}$, with which $\mathcal{J}\left(B_{z}, D_{z}\right)$ can be minimized. The formulated optimization task is:

$$
\inf _{B_{z} \in \mathbf{B}} \inf _{D_{z} \in \mathbf{D}} \mathcal{J}\left(B_{z}, D_{z}\right)
$$

It is noted that both $B_{z}$ and $D_{z}$ have bounds and they are determined by the construction of the wheel hub and the size of tire, therefore the optimization has a validity range: $\mathbf{B}=$ $\left[B_{z, \min } B_{z, \max }\right], \mathbf{D}=\left[D_{z, \min } D_{z, \max }\right]$. In the optimization task (21) different methods can be applied, e.g., [12], [13].

\section{Simulation Results}

In the example the efficiency of the integrated method based on the yaw-rate tracking scenario is presented. The vehicle is travelling along a predefined road, while the variable geometry suspension system supports the driver to guarantee trajectory tracking. In the design of the different suspension systems different construction systems and weighting strategies are applied in the control design. The first suspension system $\left(S y s_{1}\right)$ creates a balance between the different performances, the second system $\left(S y s_{2}\right)$ minimizes 
the half track change $\left(z_{5}\right.$ and $\left.z_{6}\right)$, while the third system $\left(S_{y} s_{3}\right)$ minimizes the control input $\left(z_{4}\right)$.

The vehicle is travelling along a course, which is depicted in Figure 9(a). The driver generates the front wheel steering angle as illustrated in Figure 9(b). During the maneuver the velocity of vehicle is shown in Figure 9(c). Figure 9(d) shows the tracking of the yaw rate of the vehicle with different control systems and without any control. In the uncontrolled vehicle the driver is not able to compensate for either the understeering or the oversteering motion of the vehicle, therefore the tracking error of the yaw-rate significantly increases. At the same time in all of the controlled vehicles the tracking of the reference yaw-rate with an acceptable threshold is guaranteed. Figure 9(e) shows the control inputs of the different systems, while Figure 9(f) shows the wheel camber angles. In system $\mathrm{Sys}_{3}$ the minimization of the control input $a_{y}$ is preferred, while in the other two systems larger control actuation is generated during the maneuver. However, according to the half track change the tendencies differ as it is shown in Figure $9(\mathrm{~g})$.

The performances of the yaw rate tracking are similar in all the three cases, see Figure 9(d). This result shows that acceptable tracking requires similar wheel camber angles. These camber angles are achieved in different ways: in $S_{y s}$ the actuation $a_{y}$ is minimized and the half track change is increased, while in $\mathrm{Sys}_{2}$ the minimization of the half track change is preferred besides increased actuation $a_{y}$. Figure 9(h) illustrates that the construction of the suspension system significantly influences the roll angles of chassis. This example shows that both the control design with the performance weighting strategy and the different selection of constructions influence the dynamics of the vehicle, therefore it is required to design them simultaneously in an integrated way.

\section{CONCLUSION}

The paper has proposed the simultaneous design of robust control and the construction of a variable geometry suspension system for the enhancement of vehicle stability. While the driver performs a maneuver by using the steering wheel, an autonomous control system modifies the camber angles of the rear wheels. The control design is based on robust LPV methods, in which both performance specifications and model uncertainties are taken into consideration. There is a trade-off between the control design and the variable geometry suspension construction, therefore an optimization criterium which contains both the parameters of the suspension construction and the parameters of control design is formalized.

\section{REFERENCES}

[1] R. Sharp, "Variable geometry active suspension for cars," IEEE Computing and Control Engineering Journal, vol. 9, no. 5, pp. 217 222, 1998

[2] M. S. Fallah, R. Bhat, and W. F. Xie, "New model and simulation of macpherson suspension system for ride control applications," Vehicle System Dynamics, vol. 47, no. 2, pp. 195-220, 2009.

[3] R. Sancibrian, P. Garcia, F. Viadero, A. Fernandez, and A. DeJuan, "Kinematic design of double-wishbone suspension systems using a multiobjective optimisation approach," Vehicle System Dynamics, vol. 48 , no. 7 , pp. 793-813, 2010.

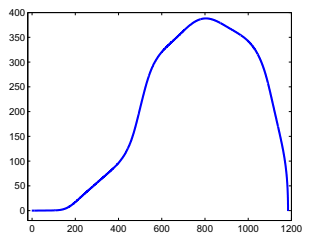

(a) Course of vehicle

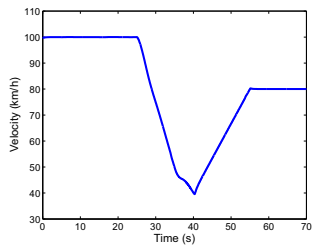

(c) Velocity of vehicle

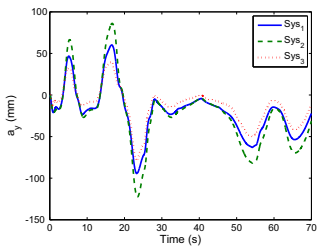

(e) $a_{y}$ input of suspension

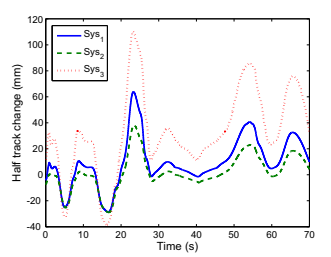

(g) Half track change

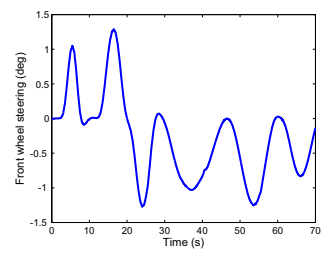

(b) Front wheel steering

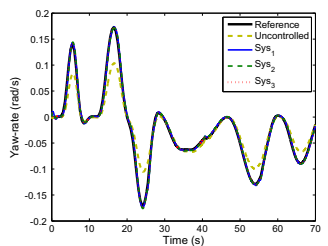

(d) Yaw-rate of vehicle

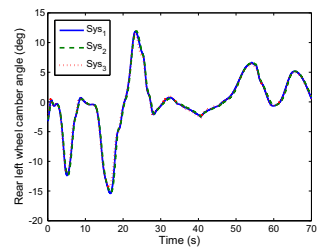

(f) Rear wheel camber

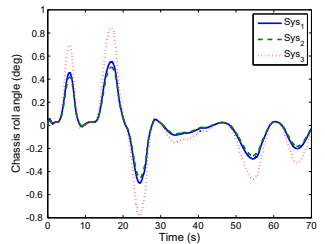

(h) Roll of chassis
Fig. 9. Analysis of suspension system

[4] M. Vukobratovic and V. Potkonjak, "Modeling and control of active systems with variable geometry. i: General approach and its application," Mechanism and Machine Theory, vol. 35, pp. 179-195, 1999.

[5] U. K. Lee and C. S. Han, "A suspension system with a variable roll centre for the improvement of vehicle handling characteristics," Proceedings of the IMechE, Part D: Journal of Automobile Engineering, vol. 215, no. 6, pp. 677-696, 2001.

[6] U. K. Lee, S. H. Lee, C. S. Han, K. Hedrick, and A. Catala, "Active geometry control suspension system for the enhancement of vehicle stability," Proceedings of the IMechE, Part D: Journal of Automobile Engineering, vol. 222, no. 6, pp. 979-988, 2008.

[7] A. Goodarzia, E. Oloomia, and E. Esmailzadehb, "Design and analysis of an intelligent controller for active geometry suspension systems," Vehicle System Dynamics, vol. 49, no. 1, pp. 333-359, 2010.

[8] J. Bokor and G. Balas, "Linear parameter varying systems: A geometric theory and applications," 16th IFAC World Congress, Prague, 2005.

[9] A. Packard and G. Balas, "Theory and application of linear parameter varying control techniques," American Control Conference, Workshop I, Albuquerque, 1997.

[10] H. B. Pacejka, Tyre and vehicle dynamics. Oxford: Elsevier Butterworth-Heinemann, 2004.

[11] R. Rajamani, "Vehicle dynamics and control," Springer, 2005.

[12] P. E. Gill, W. Murray, and M. Wright, Practical Optimization. Academic Press, London UK, 1981.

[13] T. Coleman and Y. Li, "On the convergence of reflective newton methods for large-scale nonlinear minimization subject to bounds," Mathematical Programming, vol. 64, no. 2, pp. 189-224, 1994. 